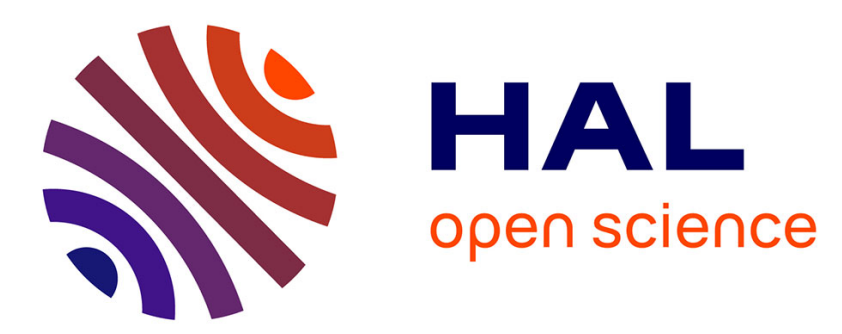

\title{
Segmented subwavelength silicon gratings manufactured by high productivity microelectronic technologies for linear to radial/azimuthal polarization conversion
}

Thomas Kämpfe, Pierre Sixt, Denis Renaud, Armelle Lagrange, Fabrice Perrin, Olivier Parriaux

\section{To cite this version:}

Thomas Kämpfe, Pierre Sixt, Denis Renaud, Armelle Lagrange, Fabrice Perrin, et al.. Segmented subwavelength silicon gratings manufactured by high productivity microelectronic technologies for linear to radial/azimuthal polarization conversion. Optical Engineering, 2014, 53 (10), pp.107105. 10.1117/1.OE.53.10.107105 . hal-01174133

\section{HAL Id: hal-01174133 \\ https://hal.science/hal-01174133}

Submitted on 10 Jul 2015

HAL is a multi-disciplinary open access archive for the deposit and dissemination of scientific research documents, whether they are published or not. The documents may come from teaching and research institutions in France or abroad, or from public or private research centers.
L'archive ouverte pluridisciplinaire HAL, est destinée au dépôt et à la diffusion de documents scientifiques de niveau recherche, publiés ou non, émanant des établissements d'enseignement et de recherche français ou étrangers, des laboratoires publics ou privés. 


\title{
Segmented subwavelength silicon gratings manufactured by high productivity microelectronic technologies for linear to radial/azimuthal polarization conversion
}

\author{
T. Kämpfe, ${ }^{a}$ P. Sixt, ${ }^{b}$ D. Renaud, ${ }^{b}$ A. Lagrange, ${ }^{b}$ F. Perrin, ${ }^{b}$ O. Parriaux ${ }^{a}$ \\ ${ }^{a}$ Université de Lyon à St-Etienne, Laboratoire Hubert Curien UMR CNRS 5516, 18 Rue du Professeur Benoît \\ Lauras, 42000 Saint-Etienne, France \\ ${ }^{b}$ Laboratoire d'électronique et de technologie de l'information CEA/Léti, 17 Rue des Martyrs, 38054 Grenoble, \\ France
}

\begin{abstract}
A polarization rotation is realized by subwavelength binary gratings, where the round trip phases of the smallest grating modes are fixed to the smallest possible integer numbers of $2 \pi$ allowing a phase difference of $\pi$ between TE and TM polarization and almost $100 \%$ transmission. The principle is applied to a polarization transformation in the 1030-1064 $\mathrm{nm}$ wavelength range, using a segmented polarization rotating element converting a linearly polarized incidence to a radial or azimuthal polarization distribution. The elevated costs of such kind of polarization transformers based on assembled birefringent crystals are avoided by using mass-fabrication compatible silicon on insulator technology on a wafer scale. It shows the general potential of microelectronic technology, concerning the batch manufacturing of wavelength-scale diffractive, grating based elements for processing free space waves.
\end{abstract}

Keywords: polarization conversion, subwavelength grating, effective index, radial polarization, azimuthal polarization, silicon on insulator.

Address all correspondence to: Thomas Kämpfe, Laboratoire Hubert Curien UMR CNRS 5516, 18 Rue du Professeur Benoît Lauras, 42000 Saint-Etienne, France; Tel: +33 477 915825; Fax: +33 477 915781; E-mail: thomas.kampfe@univ-st-etienne.fr

\section{Introduction}

Radial and azimuthal polarization distributions are interesting for diverse applications, ranging from fundamental research to practical use in industrial fabrication ${ }^{1-3}$. They can be created inside a laser resonator, for example by replacing one end-mirror with a grating having circular symmetry placed on a multilayer mirror, where one polarization component leaks into the multilayer ${ }^{4}$. The resonator will then create a donut field profile as its fundamental mode, which is not necessarily compatible with complex high power laser architectures. Therefore, there is a need for extra-cavity polarization transformers, accepting a usual linearly polarized beam and transforming it into a radial or azimuthal polarization distribution (Fig. 1). 
A known solution is an assembly of angular segments of crystal quartz with half-wave thickness, employing a different orientation of the in-plane crystal axis per segment ${ }^{5}$, which has the advantage of a high damage threshold, but is expensive and not mass fabrication compatible. The same principle can be realized also by using the form birefringence of a subwavelength binary grating of varying direction, etched into a high index substrate. However, the required small period and especially the large aspect ratio of the grooves ${ }^{6}$ are a challenge for the fabrication technology, especially if the use of mass-fabrication compatible fabrication steps is envisaged. Additionally, a high transmission for both polarizations and the required $\pi$ phase difference cannot be optimally fulfilled simultaneously by such a structure. On the contrary, subwavelength gratings using a high index grating material on a low index substrate can achieve close to $100 \%$ transmission and the required phase difference, offering the unique possibility of realizing the required polarization transformation with a reasonable aspect ratio of the grating and thus significantly relaxed fabrication technology requirements ${ }^{7,8}$. We report on the design and fabrication of such polarization transforming elements using amorphous silicon in a lithographic fabrication chain, employing planar batch processing steps developed for microelectronics in the silicon on insulator (SOI) technology. In this paper it is shown that SOI technology, which has already been applied for example for fabricating sub wavelength waveguide grating structures ${ }^{9}$, allows 8 " wafer scale mass fabrication of subwavelength grating based polarization transformers.

\section{Theory}

The structure used for the polarization transformation is a high index binary grating on a low index substrate, whose parameters are chosen so that only the fundamental TE and TM grating modes are propagating, all higher grating modes being evanescent ${ }^{7}$ (Fig. 2(a)). Using a high 
index grating on a low index substrate has four advantages with respect to a grating etched directly into a high-index substrate (example ${ }^{6}$ shown in Fig. 2(b)).

Firstly due to the larger period and much less severe aspect ratio of the grooves, the fabrication is significantly facilitated. Secondly, provided the parameters are chosen as detailed in ${ }^{7}$ so that the effective index of the fundamental TM grating mode falls between substrate and cover, an almost $100 \%$ transmission of the TM polarization is possible as the grating layer represents for this polarization an antireflection layer between the cover and the substrate. Thirdly, an almost $100 \%$ transmission is possible for the equivalent TE transmission, since the Fabry-Perot resonator for the fundamental TE grating mode can be made symmetric, i.e. the effective index of this mode can be chosen larger than both air and substrate index. And finally, choosing a relatively large period changes the phases of the reflection/transmission coefficients, which are influencing the phase round-trip conditions of the equivalent TE and TM Fabry-Perot resonances, leading to more design freedom and the possibility of fulfilling the Fabry-Perot transmission resonance for the fundamental TE mode and the TE/TM $\pi$-phaseshift condition at the same time. Additionally, it can be shown that it becomes possible to use the smallest possible integer numbers for the Fabry-Perot roundtrips to achieve transmission resonance, ${ }^{7}$ explaining the mentioned aspect ratios of $\approx 2$ (width/height) for the ridges, and $<1$ for the grooves, as compared to 4 or more in a grating etched directly into a high index substrate. It is possible to explore the grating parameter space of substrate and grating index exhaustively in order to find all possible gratings realizing the described phase compensation scheme (Fig. 2(c)). As an easy merit value the transmission through crossed polarizers with the grating being oriented at $45^{\circ}\left(T_{\text {cross }}\right)$ is used, since it will monotonically approach $100 \%$ if and only if the two conditions of $100 \%$ TE and TM transmission as well as a $\pi$-phaseshift between TE and TM are approached. As can be seen from 
Fig. 2(c) the higher the ridge index and the lower the substrate index, the better $T_{\text {cross }}$ can be optimized, with a $\approx \approx 3.5$ grating on a $n \approx 1.5$ substrate showing already $T_{\text {cross }}=97 \%$, thus pointing to SOI technology as a viable solution.

\section{Prototype fabrication by direct mask-transfer}

To demonstrate the feasibility of the proposed polarization transformer, several prototypes using a simplified laboratory technology chain (Fig. 3) were fabricated ${ }^{10}$.

The process starts by fabricating a standard chromium mask by e-beam writing. This mask was used in a hard-contact setup and transferred photolithographically into a resist layer to define the $596 \mathrm{~nm}$ period grating. Since the finally desired line width was too small to be realized directly in this step, it was reduce by wet-chemical thinning of $6 \mathrm{~min}$ in order to end up with a ridge width of about $100 \mathrm{~nm}$. Reactive ion etching with an RF-power of 50W for $100 \mathrm{~s}$ using an $\mathrm{Ar} / \mathrm{SF}_{6}$ mixture at 2 pascals was then applied to transfer the resist profile into the amorphous silicon layer down to the $\mathrm{SiO}_{2}$ surface.

A transmission SEM image of a slice of the grating cut out by a focused ion beam machine (FIB) is shown in Fig. 4(a), and the whole element under white light illumination can be seen in Fig. 4(b). The desired grating parameters of period $=616 \mathrm{~nm}$, ridge width $=109 \mathrm{~nm}$ and grating height $=250 \mathrm{~nm}$ were realized with an error of some nanometers, and the principal applicability of those elements in laser machine processing was demonstrated ${ }^{11}$.

\section{Wafer-scale, mass-fabrication compatible fabrication process}

A considerable advantage of the proposed approach of realizing segmented polarization transforming elements on the basis of subwavelength gratings is their compatibility with batch 
processing technologies established for fabricating wafer-scale micro-electronics, namely the lithographic process of structuring a silicon layer placed on insulating substrates (SOI).

We used the same design as in the case of the laboratory prototype, whose parameters were slightly adapted to period $=613 \mathrm{~nm}$, ridge width $=113 \mathrm{~nm}$ and grating height $=269 \mathrm{~nm}$ in order to account for the slightly different refractive index of the available deposited silicon of $n=3.4$. The process steps are depicted in Fig. 5. We start by a deposition of an amorphous silicon layer by the well developed process of plasma enhanced physical vapor deposition (PECVD), known from amorphous silicon solar cells production, with a thickness equal to the desired grating height. Onto the silicon we deposited a 100nm thick tetraethylorthosilicate (TEOS) hard-mask layer. Subsequently, a resist layer (JSR M78Y) of $570 \mathrm{~nm}$ thickness as well as a bottom antireflective coating (BARC) of 50nm were applied onto the hard mask layer. The grating layout was realized as a chromium reticle with a scaling of 4 by standard e-beam lithography. This reticle was then projected by means of a $248 \mathrm{~nm} \mathrm{KrF}$ stepper (ASML PAS5500/300) with a $4 \mathrm{x}$ reduction factor onto the resist layer, creating 118 single elements per wafer. The resist layer was developed and subsequently transferred into the BARC and into the hard mask layer by an inductively coupled plasma etching processes (ICP), using a $\mathrm{CF}_{4} / \mathrm{O}_{2} / \mathrm{Ar}$ mixture for 20 s to etch the $\mathrm{BARC}$ and a $\mathrm{C}_{4} \mathrm{~F}_{8} / \mathrm{O}_{2} / \mathrm{CO} / \mathrm{Ar}$ mixture for $17 \mathrm{~s}$ to transfer into the hard mask. Since the ridge width of the polarization transformer is too small to be realized directly, a wet-etching process using a $1 \% \mathrm{HF}$ solution for $90 \mathrm{~s}$ is applied, reducing the ridges isotropically by $40 \mathrm{~nm}$ in order to trim the line width down to the required $113 \mathrm{~nm}$. Finally, the hard-mask is transferred to the amorphous silicon layer in a highly selective ICP etching process using a $\mathrm{Cl}_{2} / \mathrm{HBr}$ mixture for $75 \mathrm{~s}$. The remnants of the hard mask, having a height of about $30 \mathrm{~nm}$, are subsequently removed 
via a $\mathrm{C}_{4} \mathrm{~F}_{8} / \mathrm{O}_{2} / \mathrm{CO} / \mathrm{Ar}$ ICP etching step of $10 \mathrm{~s}$, where the length of this step is optimized in order to completely remove the remaining mask.

The resulting gratings were analyzed in a FIB, showing a clean line profile (Fig. 6) with some deviations from the design: The height of the grating is smaller than the design, which is due to the removal process of the hard mask, that was slightly too long. A considerable overetch into the substrate of about $40 \mathrm{~nm}$ can also be seen, which is due to the hard mask removal being a non-selective process, i.e. attacking also the substrate. Finally the shape of the upper part of the lines is slightly trapezoidal, which is a sign for beginning mask degradation during the aSietching. All of those effects can be reduced by carefully optimizing the process. The effects of the changed grating parameters on the optical functionality will be detailed in the following chapter. All mentioned deviations are to be expected as part of the calibration of the SOI process chain, since the range of the parameters are different from better controlled microelectronics applications. The errors will be significantly reduced in further optimization steps

\section{Characterization and optical testing}

The optical characterization of the elements was done using a collimated Nd:Yag laser with $1064 \mathrm{~nm}$ wavelength (Fig. 7(a)). The beam diameter was reduced via a telescope to $0.2 \mathrm{~mm}$ diameter in order to be able to measure the outer parts of the angular grating segments separately, in order to measure the transmission through crossed polarizers $T_{\text {cross, }}$, where the grating is oriented at $45^{\circ}$ with regard to the direction of the incoming polarization. For the laboratory prototypes, a transmission of more than $97 \%$ for both polarizations could be measured. $T_{\text {cross }}$ reached in some segments a maximum value of $95 \%$, with a theoretical maximum of $96.2 \%$. The average over all segments of $\mathrm{T}_{\text {cross }}$ was measured to be $90 \%$. The lower transmission in several sectors can be attributed to slight differences in the realized grating 
parameters, as well as to scattering due to a remaining line roughness that depends on the local orientation of the lines due to their definition in a shape-base e-beam machine. The efficient creation of radially polarized light was demonstrated by analyzing the intensity distribution of a widened beam (adapted to the element size of $6 \mathrm{~mm}$ ) which after passing through the element was filtered by an additional linear polarizer. The output intensity distribution shows the typical butterfly shape, indicating the presence of radial polarization. First tests to determine the laser flux resistance were made in a setup with a femtosecond Yb:YAG laser, leading to the main conclusion that the damage threshold of the polarization transformer in this case was close to the usual operation conditions of the machine ${ }^{11}$, proving that an operation without additional beam diameter expansion is feasible.

The measurement of $T_{\text {cross }}$ for a wafer of 118 elements fabricated by the process depicted in Fig. 5 is shown in Fig. 7(b). The layout in the $\mathrm{Cr}$ reticle was chosen to realize a variation of the line width in order to tune the process. In order to do this the line width in the $\mathrm{Cr}$ reticle for the 4 gratings arranged in a $2 \times 2$ layout as shown in the upper left corner by the dotted square in Fig. 7(b) were chosen to be $180,200,220$ and $240 \mathrm{~nm}$ for the elements 1 to 4 respectively, thus allowing to find a grating with the correct line width of $113 \mathrm{~nm}$ after completing all process steps. This $2 \times 2$ layout was repeated over the whole wafer without additional changes in order to judge the overall homogeneity of the process. The results shown in Fig. 7(b) lead to the following conclusions: Firstly the polarization transformation could be verified but with a significantly lower transmission than in the case of the laboratory prototype. The Cr reticle grating line width which is best adapted is found to be $220 \mathrm{~nm}$ in quadrant 3 . Additionally the elements situated more towards the center of the wafer show a better performance. The FIB image of one of those elements is shown in Fig. 6(b). A simulation made with the measured line width, grating height 
and overetch, including the trapezoidal line shape at the top, resulted in a theoretical value for $T_{\text {cross }}$ of $82 \%$, while the measurement for this element remained at about $T_{\text {cross }}=76 \%$. The difference is probably caused mainly by scattering losses due to particles sticking to the sidewalls of the lines (see SEM top view in Fig. 6(b)). Secondly there is a systematic variation

over the wafer were the best elements are found in the middle. This is confirmed by the photograph in Fig. 6(a) where additionally a circular region of defective elements shows that there is some global, circularly symmetric variation of the process parameters, most probably connected to the layer deposition processes.

\section{CONCLUSION}

We demonstrated the design, fabrication and application of polarization transformers based on subwavelength gratings, made in a hydrogenated amorphous silicon layer on a low index substrate. We showed that the use of a low index substrate provides substantial advantages in comparison with a grating directly etched into the substrate. The optical functionality was demonstrated showing a good agreement with the theoretical calculations. To demonstrate the compatibility with mass fabrication technology several 8" wafers carrying 118 elements were fabricated, showing a less optimal optical performance due to deviation of the shape and parameters of the grating lines, caused by a not yet sufficiently optimized process chain. Beyond the realization of this particular polarization-rotating element, this work aims at presenting the potential of mass-fabrication compatible lithographic processes for microelectronics fabrication in the context of batch manufacturing of diffractive optical elements with wavelength scale features for the processing of free space waves.

\section{Acknowledgments}


The authors would like to acknowledge the financial support by the region Rhône-Alpes within the CMIRA-grant 1200618901.

\section{References}

1. R. Weber, A. Michalowski, M. A. Ahmed, V. Onuseit, V. Rominger, M. Kraus, and T. Grafal, "Effects of Radial and Tangential Polarization in Laser Material Processing," Phys. Procedia 12 , 21$30(2011)$

2. T. Nieminen, N. Heckenberg, and H. Rubinsztein-Dunlop, "Forces in optical tweezers with radially and azimuthally polarized trapping beams," Opt. Lett. 33, 122-124 (2008).

3. R. Dorn, S. Quabis, and G. Leuchs, "Sharper Focus for a Radially Polarized Light Beam” Phys. Rev. Lett. 91, 233901 (2003)

4. M. A. Ahmed, J. Schulz, A. Voss, O. Parriaux, J. C. Pommier, and T. Graf, "Radially polarized 3 kW beam from a CO2 laser with an intracavity resonant grating mirror," Opt. Lett. 32, 1824-1826 (2007)

5. M. Kraus, M. A. Ahmed, A. Michalowski, A. Voss, R. Weber, and T. Graf, "Microdrilling in steel using ultrashort pulsed laser beams with radial and azimuthal polarization," Opt. Express 18, 22305$22313(2010)$

6. G. M. Lerman and U. Levy, "Generation of a radially polarized light beam using space-variant subwavelength gratings at $1064 \mathrm{~nm}, "$ Opt. Lett. 33, 2782-2784 (2008)

7. T. Kämpfe and O. Parriaux, "Depth-minimized, large period half-wave corrugation for linear to radial and azimuthal polarization transformation by grating-mode phase management," J. Opt. Soc. Am. A 28, 2235-2242 (2011).

8. O. Parriaux and T. Kämpfe, „Planar grating polarization transformer“, EP 2530499 A1 (2012)

9. K. J. Lee, R. LaComb, B. Britton, M. Shokooh-Saremi, H. Silva, E. Donkor, Y. Ding and R. Magnusson, "Silicon-layer guided-mode resonance polarizer with 40-nm bandwidth," IEEE Photonics Technology Letters 20, 1857-1859 (2008). 
10. T. Kaempfe, S. Tonchev, G. Gomard, C. Seassal and O. Parriaux, "Hydrogenated amorphous silicon microstructuring for 0 th order polarization elements at $1.0 \mu \mathrm{m}-1.1 \mu \mathrm{m}$ wavelength," IEEE Photonics J. 3, 1142-1148 (2011).

11. R. Torres, T. Kaempfe, M. Delaigue, O. Parriaux, C. Hönninger, J. Lopez, R. Kling and E. Mottay, "Influence of laser beam polarization on laser micro-maching," 13th International Symposium on Laser Precision Microfabrication (2012)

\section{Biographies}

Thomas Kämpfe: Thomas Kämpfe received his diploma in physics in 2004 from the FriedrichSchiller-University Jena, Germany. In 2009 he finished his $\mathrm{PhD}$ at the Institute of Applied Physics, also in Jena. He works now at the Laboratoire Hubert Curien of the University of Lyon as teacher and researcher in the field of design and fabrication of diffractive micro-optical elements, especially in the area of resonant gratings, polarizers and computer generated holograms.

Armelle Lagrange: After a master degree in Semiconductor Physics at the Institut National Polytechnique in Grenoble in 1993, Armelle Lagrange gained her PhD in 1996 at the CEA-LETI. Her $\mathrm{PhD}$ dissertation was on optoelectronics devices (laser waveguides). After working at ST Microelectronics as a lithography process engineer, she joined CEA-LETI-MINATEC as a research engineer in lithography in 2008.

Pierre Sixt: Pierre Sixt received his $\mathrm{PhD}$ in Optoelectronics from Grenoble's Institut National Polytechnique in 1989. After working on optical sensors development and e-beam microfabrication at CSEM in Neuchâtel, Switzerland, he joined the global mask maker Photronics in 1996 where he participated in the simulation, process and production of advanced 
reticles. Since 2006, he is leading $200 \mathrm{~mm}$ lithography process development activities at CEALeti.

\section{$\underline{\text { Figure captions list }}$}

Fig. 1 Transformation of linear to radial polarization by a subwavelength grating creating a $\pi$ phase shift between TE and TM polarization.

Fig. 2 Polarization transforming grating, (a) using a high index layer on a low index substrate, and (b) directly etched into a high index substrate. The applicability of the principle depicted in (a) for different substrate $\left(\mathrm{n}_{\mathrm{s}}\right)$ and grating $\left(\mathrm{n}_{\mathrm{g}}\right)$ materials using $T_{\text {cross }}$ (see text) as merit value is shown in (c)

Fig. 3 Fabrication steps for a flexible production of laboratory prototype by direct mask-transfer.

Fig. 4 (a) SEM-image of the cross-section of the grating (extracted by FIB machining) of a laboratory prototype, (b) photograph of the prototype under white light.

Fig. 5 steps of the step and repeat, 8" wafer-scale, mass-fabrication compatible fabrication process.

Fig. 6 (a) photograph of a 8" wafer with 118 polarization transforming elements, (b) SEM images of the grating lines and their cross-section prepared by FIB machining.

Fig. 7 (a) Optical measurement setup for determining $T_{\text {cross }}$ (definition see text), (b) results for a 8 " wafer with 118 elements (layout details see text). 


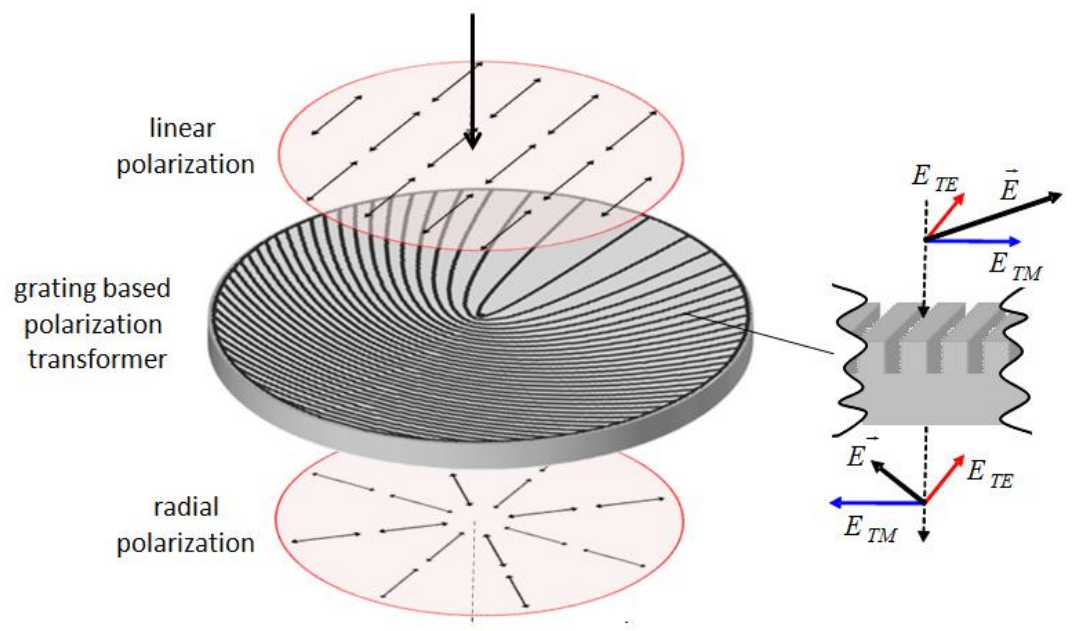

Fig. 1 Transformation of linear to radial polarization by a subwavelength grating creating a $\pi$-phase shift between TE and TM polarization.

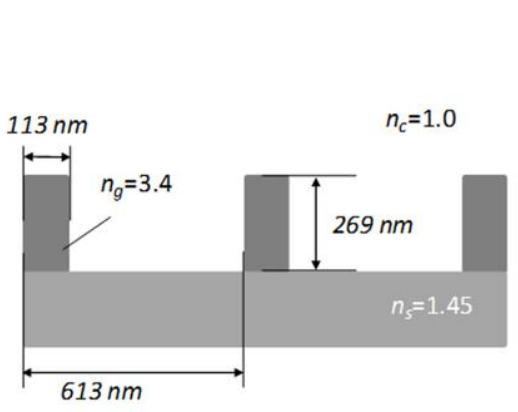

(a)

(a)

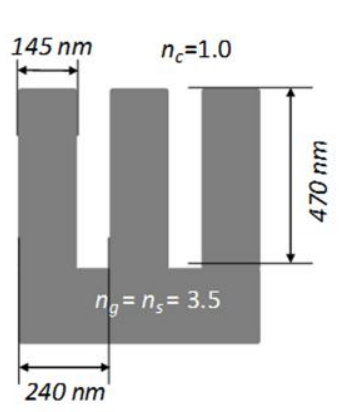

(b) (b)

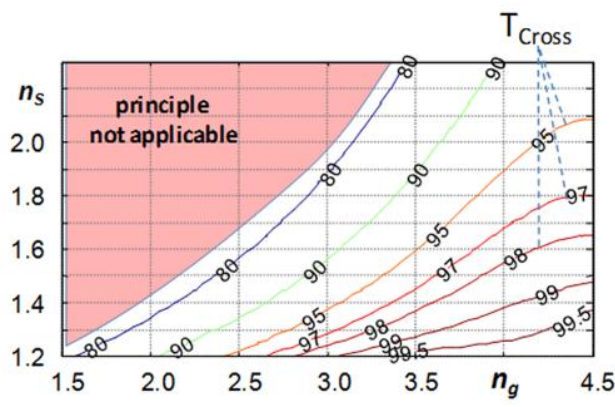

(c)

Fig. 2 Polarization transforming grating directly etched into a high index substrate (a), and using a high index layer on a low index substrate (b). The applicability of the principle depicted in (b) for different substrate $\left(\mathrm{n}_{\mathrm{s}}\right)$ and grating $\left(\mathrm{n}_{\mathrm{g}}\right)$ materials using $T_{\text {cross }}$ (see text) as merit value is shown in (c) 


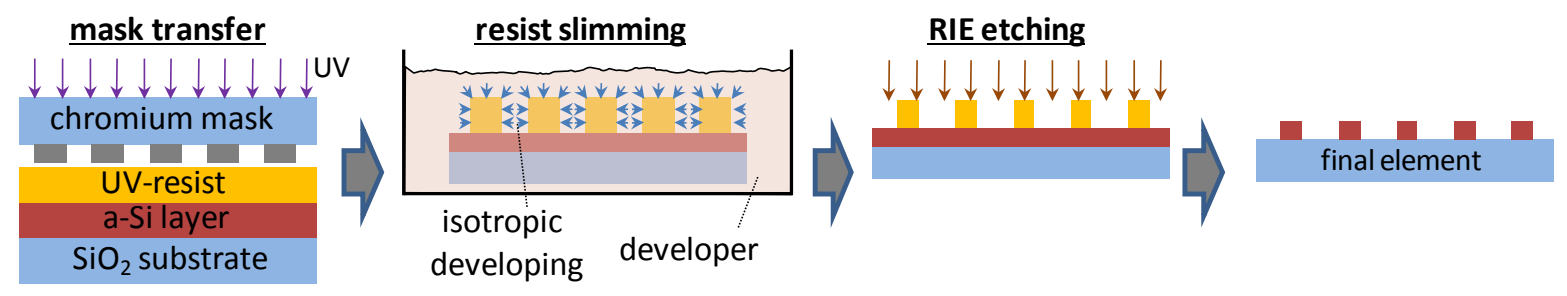

Fig. 3 Fabrication steps for a flexible production of laboratory prototype by direct mask-transfer.

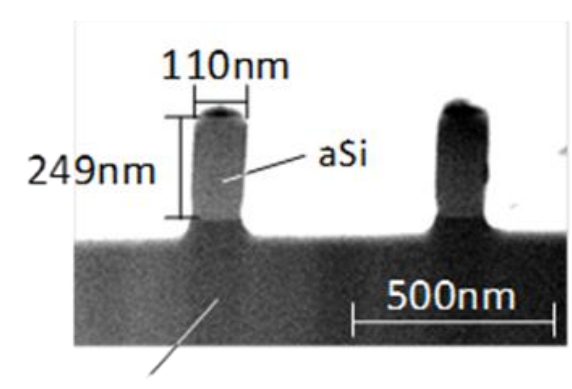

$\mathrm{SiO}_{2}$ substrate

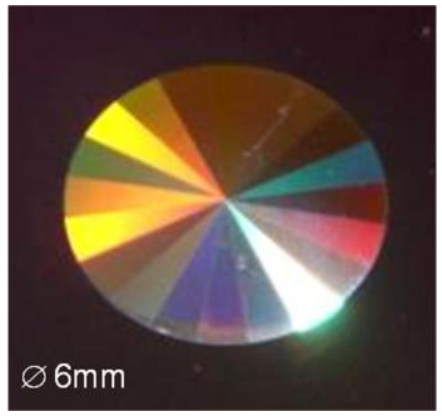

(b)

Fig. 4 (a) SEM-image of the cross-section of the grating (extracted by FIB machining) of a laboratory prototype, (b) photograph of the prototype under white light.

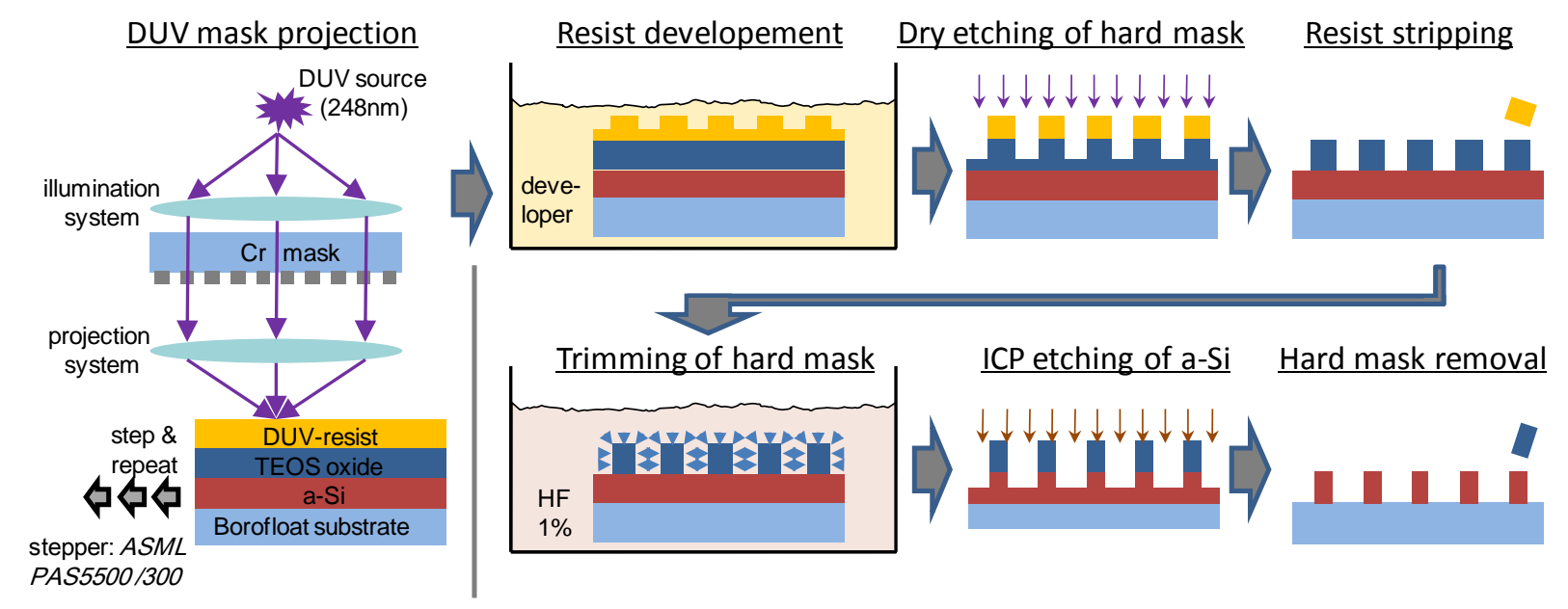

Fig. 5 Fabrication steps of the step and repeat, 8" wafer-scale, mass-fabrication compatible fabrication process. 

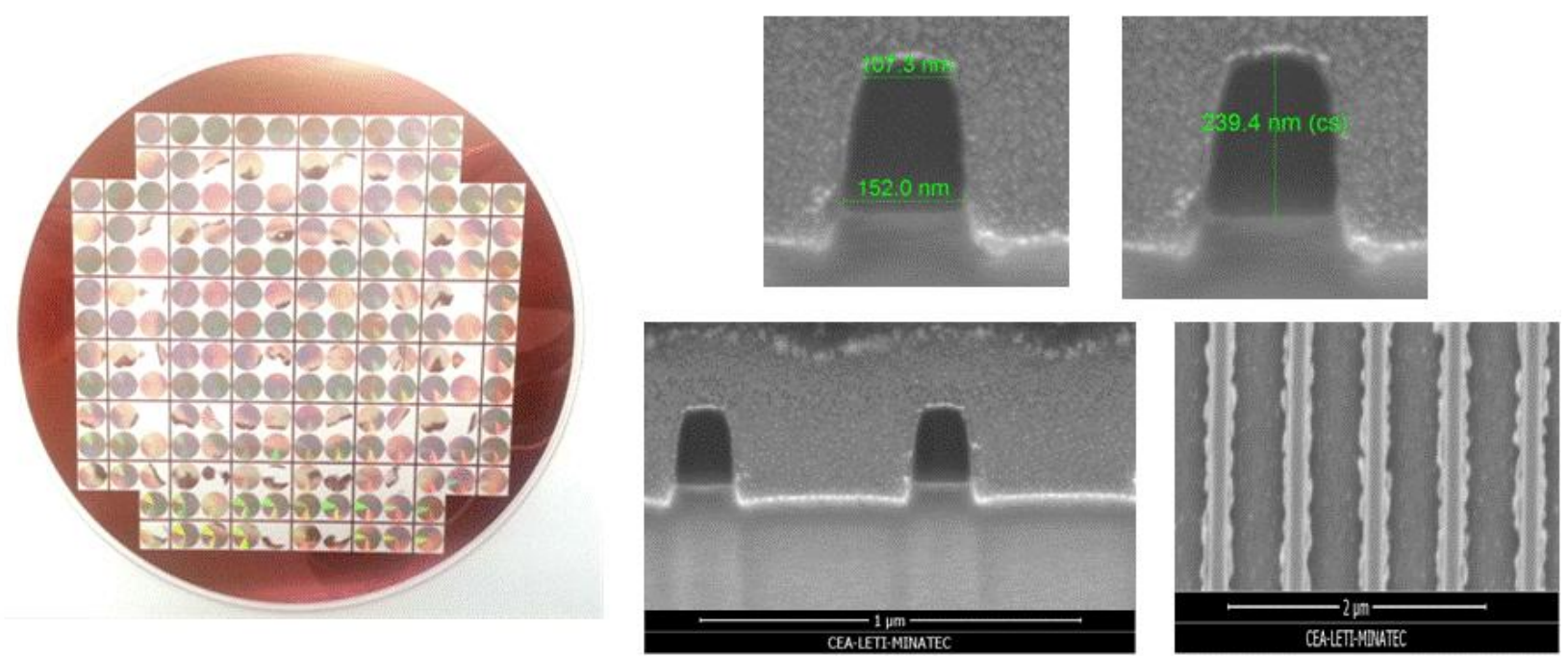

Fig. 6 (a) photograph of a 8" wafer with 118 polarization transforming elements, (b) SEM images of the grating lines and their cross-section prepared by FIB machining.

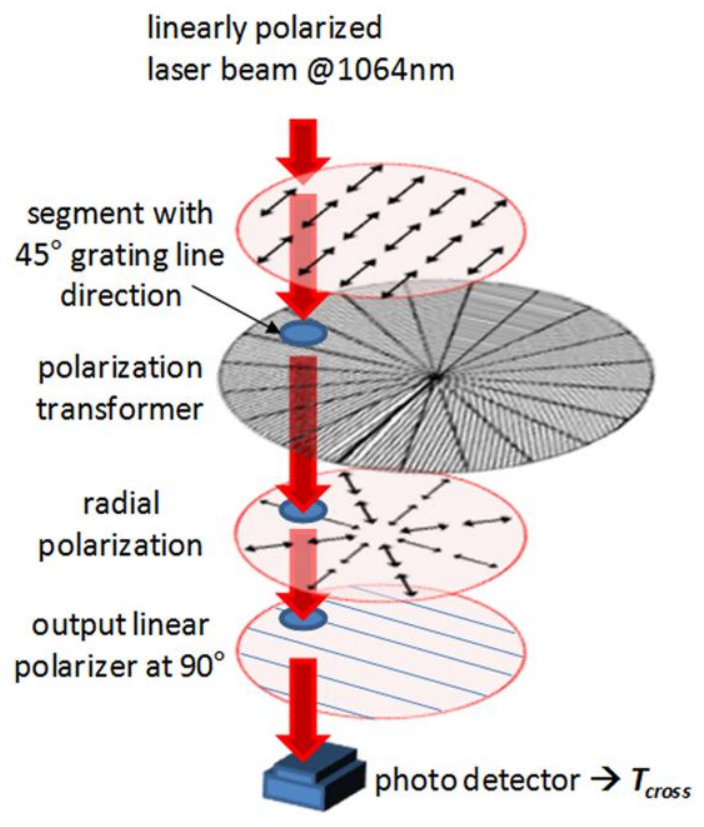

(a)

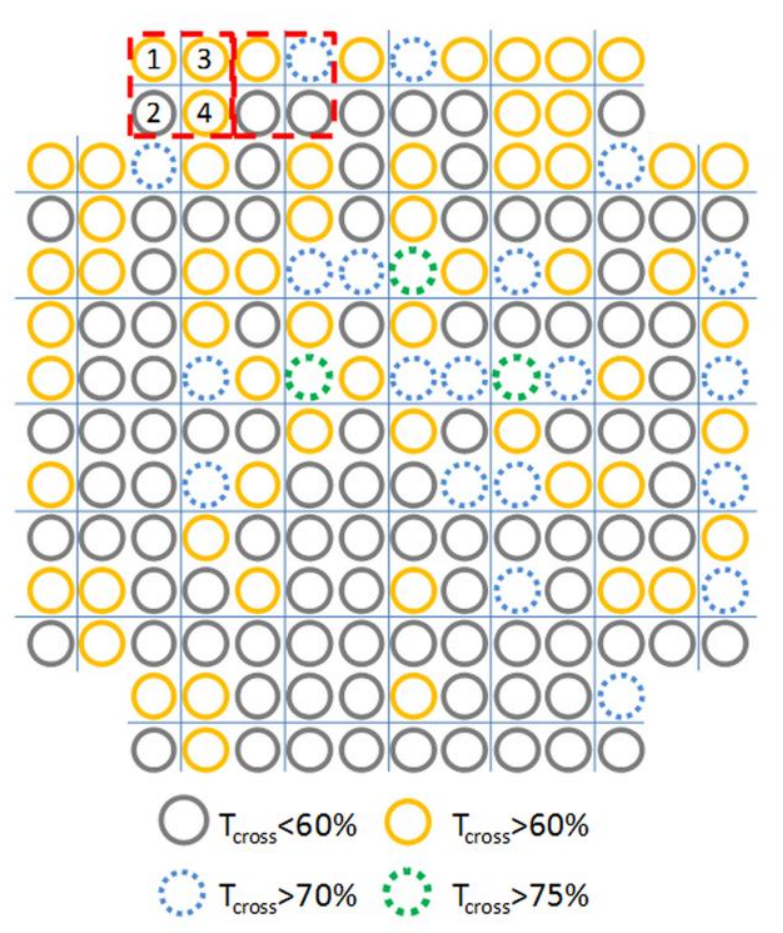

(b)

Fig. 7 (a) Optical measurement setup for determining $T_{\text {cross }}$ (definition see text), (b) results for a 8 " wafer with 118 elements (layout details see text). 
